\title{
Le radicalisme anglais et la Révolution française
}

\section{Michael Davis}

\section{(2) OpenEdition}

\section{Journals}

Édition électronique

URL : https://journals.openedition.org/ahrf/1913

DOI : 10.4000/ahrf.1913

ISSN : 1952-403X

Éditeur :

Armand Colin, Société des études robespierristes

Édition imprimée

Date de publication : 1 décembre 2005

ISSN : 0003-4436

Référence électronique

Michael Davis, "Le radicalisme anglais et la Révolution française ", Annales historiques de la Révolution française [En ligne], 342 I octobre-décembre 2005, mis en ligne le 16 septembre 2013, consulté le 22 avril 2022. URL : http://journals.openedition.org/ahrf/1913 ; DOI : https://doi.org/10.4000/ahrf.1913

Ce document a été généré automatiquement le 22 avril 2022

Tous droits réservés 


\title{
Le radicalisme anglais et la Révolution française
}

\author{
Michael Davis
}

1 «Chaque Européen prend aujourd'hui part à ce dernier conflit de civilisation [...]. En étant cosmopolitique pour ainsi dire, la Révolution cesse d'appartenir seulement aux Français " ${ }^{1}$. Ainsi écrivait Jacques Mallet du Pan en 1793, alors que la Révolution française était entrée dans sa phase régicide sous Robespierre, et qu'une guerre sanglante déchirait l'Europe. Peu, parmi les contemporains, auraient nié l'importance et la profondeur des conséquences de la Révolution française, à mesure qu'elle progressait dans un kaléidoscope de changements. Encore plus rares étaient ceux qui ne furent pas affectés par son influence omniprésente. Les échos venus de France étaient particulièrement vibrants en Grande-Bretagne, polarisant les appartenances idéologiques à un degré sans précédent. Toutefois, selon Clive Emsley, les événements de 1789 qui déclenchèrent la Révolution française «ne suscitèrent que peu d'agitation ou d'enthousiasme en Grande-Bretagne » ${ }^{2}$. Pour beaucoup de Britanniques, 1789 fut plus marquée par la guérison temporaire de George III que par les événements d'OutreManche. Pourtant, les historiens de l'époque de Thomas Carlyle ont identifié et analysé la manière dont les influences de la Révolution française ont touché la GrandeBretagne ${ }^{3}$.

2 D'après Hedva Ben-Israel, «en Angleterre, plus même qu'en France, on écrivait l'histoire de la Révolution française avant que celle-ci ne soit achevée " ${ }^{4}$. Ceux qui, dans sa phase initiale, louèrent la Révolution le firent alors qu'elle ne s'était pas encore dévoilée entièrement. Quand Charles James Fox décrivit la Révolution, en 1789, comme "l'événement le plus important [...] qui soit jamais arrivé dans le monde " 5 , il exprimait une opinion commune, prédominante parmi les Whigs de l'époque, qui voyait la Révolution française comme une reconstitution tardive et opportune de 1688. À mesure que les nouvelles de France commençaient à filtrer dans la presse anglaise et dans les correspondances privées à partir du milieu de 1792, les perceptions de la Révolution se modifièrent peu à peu : d'une Glorieuse Révolution française, on passa à 
la vision d'un gouvernement républicain qui n'était pas sans rappeler la GrandeBretagne du milieu du XVII ${ }^{\mathrm{e}}$ siècle.

3 Il est clair que la nouvelle des sanglants massacres de septembre et de l'exécution de la famille royale en France douchèrent l'enthousiasme anglais pour la Révolution. Cependant, c'est seulement lorsque ce que l'on appelait la « maladie française » sembla devoir contaminer la Grande-Bretagne qu'une hostilité prononcée et concertée envers les sympathisants des Français se mit à enfler. Dès l'origine, on proclama en France que la Révolution était une bénédiction que l'on voulait exporter à d'autres nations, « une nouvelle croisade, une croisade pour la liberté universelle $»^{6}$. En imposant des allégeances internationales par une politique étrangère délibérément agressive et résonnante, la France chercha à encourager les autres nations à fonder leurs propres républiques sœurs, dédiées aux mêmes principes et en alliance avec elle ${ }^{7}$. Les réformateurs anglais, encouragés par ces sentiments fraternels, soutenaient l'idée que les Français «ne revendiquent pas uniquement leur propres droits, mais font avancer les libertés universelles du genre humain ${ }^{8}$. Lorsque la guerre éclata entre la GrandeBretagne et la France en février 1793, le départ en croisade affiché par les Français et la rhétorique fraternelle des réformateurs anglais prirent une signification nouvelle. Il devint vital pour l'État britannique « de supprimer à sa source le cancer des principes révolutionnaires ${ }^{9}$.

4 Cette guerre mit en lumière et élargit la coupure entre les partisans et les détracteurs de la Révolution. Dans ces années-là, comme l'a remarqué J.G.A. Pocock, «il devint important pour certains de faire valoir, et pour d'autres de contester, le droit de penser son propre pays dans son tort, et même de souhaiter sa défaite dans une cause injuste » ${ }^{10}$. Le patriotisme, la francophobie et le conservatisme distinguèrent les loyalistes de ceux que l'on stigmatisa collectivement sous le terme de jacobins anglais, considérés comme des éléments subversifs et des traittres. Milan Kundera a même suggéré que « depuis l'époque de la Révolution française, une moitié de l'Europe s'intitule la gauche, et l'autre moitié a reçu l'appellation de droite " ${ }^{11}$. À l'appui de ce point de vue, George Rudé avait montré qu'un « résultat précoce de la Révolution française fut de diviser la société européenne en deux groupes distincts et mutuellement hostiles - les partisans des "patriotes" d'un côté, et leurs opposants ou "contre-révolutionnaires" de l'autre " ${ }^{12}$. La polarisation de l'opinion publique de part et d'autre d'une frontière loyaliste / radicale fut accentuée par la guerre idéologique qui se cristallisa autour du débat pamphlétaire entre Edmund Burke et Thomas Paine. Sur les rives opposées du golfe politique créé par la Révolution française, Burke et Paine se dressaient comme des «boxeurs intellectuels ${ }^{13}$.

5 Les chercheurs contemporains ont poursuivi, dans une certaine mesure, ce pugilat intellectuel. Il est certain que le patriotisme et l'anti-jacobinisme étaient des sentiments bien plus présents dans la société anglaise de la fin du XVIII ${ }^{e}$ siècle que le radicalisme. Ceci explique que le loyalisme et le conservatisme dans les années 1790 aient fait l'objet de nombreuses études récentes ${ }^{14}$, conduisant un auteur à affirmer : « Il semble presque que les héritiers de Burke ont totalement chassé les héritiers de Paine des pages de l'historiographie ${ }^{15}$. Cet article, toutefois, montrera que les « héritiers de Paine» restent vivants et actifs. À travers une étude des publications récentes, on montrera qu'au plan de la recherche, le radicalisme anglais des années 1790 est un champ bien arpenté, dynamique et plein de vitalité ${ }^{16}$. 


\section{Les sources}

6 Les radicaux anglais de la fin du XVIII ${ }^{\mathrm{e}}$ siècle vivaient dans un monde où les discours étaient riches et divers. La recherche a récemment fait d'immenses progrès en mettant à jour et en reproduisant les productions imprimées et manuscrites de la période. Sans surprise, une partie significative de ces projets éditoriaux a été consacrée à des travaux produits par ce que l'on a appelé la "guerre des pamphlets» ${ }^{17}$, autour de la controverse Burke / Paine ${ }^{18}$. D'après Thomas Copeland, cet échange fut «sans doute le débat idéologique le plus crucial jamais soutenu en anglais " ${ }^{19}$, tandis qu'Alfred Cobban considère qu'il s'agissait de

«la dernière discussion réelle sur les principes essentiels de la politique [...]. Des problèmes aussi importants ont été soulevés de notre temps, mais il serait faux de prétendre qu'ils ont suscité une discussion politique de la même tenue intellectuelle que ceux qu'inspira la Révolution française. ${ }^{20}$

7 Le débat commença avec la publication des Réflexions sur la Révolution en France (1790), réponse directe à un sermon de Richard Price devant la Revolution Society en novembre 1789. Les réponses à Burke ne tardèrent pas à arriver, émanant d'auteurs comme James Mackintosh, Capel Lofft, Joseph Priestley, Joseph Towers et Mary Wollstonecraft ${ }^{21}$. Ce fut toutefois Thomas Paine qui offrit la réponse la plus pertinente dans les Droits de l'homme, publié en deux parties en mars 1791 et en février 1792.

Dans les résumés contemporains de ces débats, on trouve généralement des extraits de Paine, de Burke et d'une sélection d'auteurs représentatifs. Dans l'ouvrage de M. Butler ${ }^{22}$, et plus récemment dans les volumes de G. Claeys ${ }^{23}$ et I. Hampsher-Monk ${ }^{24}$, l'impact idéologique de la Révolution française est présenté pour la majeure partie à travers des sélections des œuvres de E. Burke, Th. Paine, R. Price, M. Wollstonecraft et W. Godwin. Si ces anthologies fournissent des introductions utiles et une vue d'ensemble des enjeux plus vastes de la guerre pamphlétaire, leur contenu est dicté par le pouvoir discrétionnaire des éditeurs. Or la confrontation avec les textes intégraux est le seul moyen de se familiariser pleinement avec le contexte et la pensée de ces auteurs : sur ce point aussi, la recherche récente a fait de remarquables progrès. À cet égard, la publication des huit volumes des Political Writings of the $1790 \mathrm{~s}{ }^{25}$ marque un jalon important. Les travaux les plus connus de Paine et Burke n'y sont pas reproduits, mais on y trouve à la place une anthologie éclectique de pamphlets des années 1790, dont beaucoup sont des textes rares et peu connus. Chaque texte est reproduit intégralement et recomposé, tandis que l'ordonnancement des volumes suit, fort utilement, une logique à la fois chronologique et thématique. L'ensemble est muni d'une instructive introduction de 40 pages.

9 La recomposition des textes de l'édition des Political Writings of the 1790s écarte tout contrôle de qualité permis par une publication en fac-similé, et pour les chercheurs désireux d'un contact plus authentique avec les originaux, la Eighteenth Century Collection Online publiée par Thomson Gale constitue l'une des meilleures ressources disponibles. On trouve en effet dans ce corpus tous les longs pamphlets radicaux des années 1790, sous forme de fac-similé. Issue de la Eighteenth Century Microfilm Collection, cette base de données contient un total de 150000 œuvres, ce qui en fait l'une des plus riches et des plus complètes archive historique de ce type en ligne. En revanche, ce qui y manque est l'appareil critique que l'on trouve dans les reproductions imprimées. 
10 Par chance, nous ne manquons pas d'éditions complètes imprimées des écrits radicaux à partir des années 1790. Il n'est guère surprenant que les écrits de Thomas Paine aient été populaires pour les éditions universitaires, au point de créer presque une saturation du marché. Les premières éditions des Droits de l'homme atteignirent, en effet, des chiffres impressionnants. Horst Ihde considère même que « durant la vie de l'auteur, plus d'un million et demi de copies furent imprimées et vendues " ${ }^{26}$. L'intérêt pour le pamphlet séminal de Paine ne semble pas se tarir aujourd'hui. Il n'existe pas moins de trois éditions complètes des Droits de l'homme $e^{27}$, ainsi que quatre recueils des œuvres de Paine ${ }^{28}$. Ces derniers reproduisent aussi la première partie de L'Âge de raison (1794), une attaque imprégnée de déisme contre la place de la religion dans la société, qui fut donnée à Joel Barlow, sous forme de manuscrit, sur le chemin qui le menait à la prison en décembre 1793. Deux versions éditées de ce pamphlet sont également disponibles séparément ${ }^{29}$. Le recueil de référence sur les œuvres de Paine, englobant la période 1774 à 1804, parut d'abord en 1894, par Moncure Daniel Conway; il a également été réimprimé. L'une des avancées les plus récentes à ce sujet est la sortie d'un CD-ROM sous le titre An Appreciation of Thomas Paine, qui contient 27 livres et revues intégralement reproduits ${ }^{30}$.

11 Le progrès du multimédia s'est révélé toujours plus payant pour les chercheurs intéressés par le radicalisme anglais des années 1790, avec la parution de The State Trials on CD-ROM ${ }^{31}$. Pour la majeure partie, il comprend les 33 volumes des State Trials de Howell ${ }^{32}$, où l'on trouve des comptes rendus in extenso des procès pour sédition et trahison des années 1790. Il s'agit d'une ressource formidable pour les spécialistes d'histoire politique et d'histoire du droit, avec tout l'apport de la numérisation, et notamment les fonctions « rechercher » et « liens » : de la sorte, il n'est plus nécessaire de consulter les grands volumes imprimés poussiéreux et souvent fragiles. Les éditions électroniques ont également remplacé d'autres volumes moisis et à la consultation peu commode: ceux de la British Library's Additional Manuscripts, et en particulier les archives de la London Corresponding Society (LCS). Celle-ci, fondée en 1792, devint rapidement la principale société radicale de la période. L'un de ses leaders, Francis Place, avait collationné la plupart des documents que l'on trouve à la British Library, que l'éditeur Microform Academic Publishers a récemment reproduits sur CD-ROM ${ }^{33}$.

12 Le matériau que l'on trouve sur ce CD-ROM sera familier pour ceux qui ont lu les Selections from the Papers of the London Corresponding Society ${ }^{34}$, dans lesquelles Mary Thale avait édité avec une grande finesse une sélection de textes imprimés et d'archives concernant la LCS. Des annotations approfondies sont ainsi fournies dans tout le recueil, ce qui donne au lecteur un aperçu du monde des sociétés réformatrices de la fin du XVIII ${ }^{e}$ siècle, principalement masculin. On y découvre que la LCS était infiltrée par des espions à la solde du gouvernement et des informateurs perfides, et que la menace de poursuites judiciaires n'était jamais bien loin. Ces archives mettent au jour un groupe souvent déchiré par les conflits internes, en particulier à la fin des années 1790, lorsque les partisans du coup de force commencèrent à dominer la hiérarchie de la société. C'est une autre facette de la LCS qui émerge avec la publication récente, en six volumes, des imprimés de la société ${ }^{35}$. Avec une unité presque parfaite, on y voit la société proclamer sa solidarité et son programme de réforme modéré dans une campagne écrite qui visait d'abord à se justifier et à se légitimer publiquement, mais aussi à éclairer politiquement les classes laborieuses. 

Lumières, est renforcée dans l'autobiographie et les mémoires de deux des principaux membres de la LCS : Francis Place et Thomas Hardy ${ }^{36}$. Pour ces deux hommes, la LCS était la source d'un progrès moral et politique. Leurs écrits permettent de comprendre qu'ils se battaient autant pour l'éducation et la civilité populaire que pour la réforme démocratique : la réussite de la seconde dépendait du succès préalable des premières. Beaucoup de sources soutiennent la thèse d'une centralité de l'esprit didactique dans le mouvement radical des années 1790. En 1796 par exemple, John Binns et John Gale Jones furent nommés députés de la LCS pour visiter les centres régionaux de l'Angleterre. E.P. Thompson discerne une intention révolutionnaire dans ces périples ${ }^{37}$. Le récit de voyages de Gale Jones dans le North Kent, qui a récemment fait l'objet d'une réédition ${ }^{38}$, indique que l'équipée avait en fait pour but d'évaluer l'importance de l'activité politique dans la région, et de promouvoir le programme de la réforme par le biais du débat. De façon similaire, pour John Thelwall, intellectuel jacobin de premier plan, membre de la LCS et orateur doué, le gros de la campagne réformatrice était centré sur l'éducation, en recourant à la prose et aux discours. La lecture de The Politics of English Jacobinism ${ }^{39}$ permet de saisir le sens de ce programme éducatif à travers le discours spécifiquement jacobin de Thelwall ${ }^{40}$.

Thelwall était doté de la capacité d'exprimer ses idées politiques et sociales directement à un public plébéien, à l'instar de Thomas Spence, membre occasionnel de la LCS et propagandiste radical prolifique dans les années 1790. Spence se faisait l'avocat de la révolution politique et sociale, et de façon plus controversée défendait une redistribution de la propriété dans ce qui était connu comme son «projet terrien» (Land Plan). Les éditions de ses écrits, par Dickinson et Gallop, donnent accès à ses œuvres politiques les plus importantes à partir des années 1790, mais son hebdomadaire à succès, Pig's Meat, ne bénéficie toujours pas d'une (ré)édition moderne ${ }^{41}$. Néanmoins, l'ajout de poèmes et de chansons dans l'édition de Dickinson donne une idée des multiples genres [littéraires] auxquels recourait Spence pour transmettre ses messages radicaux. Cette diversité du programme de publication radicale est à l'œuvre dans un ouvrage édité par Michael Scrivener, Poetry and Reform. Periodical Verse from the English Democratic Press 1792-1824 ${ }^{42}$, qui inclut une sélection de vers de journaux et de revues, y compris le Pig's Meat de Spence, pour illustrer la rébellion formelle des poètes démocrates dans les années 1790. Dans une veine similaire, John Barrell a collecté 26 ballades satiriques du milieu des années 1790 qui raillent l'État et la monarchie avec humour mais aussi efficacité ${ }^{43}$.

Les ballades et la poésie ne sont qu'un aspect de la riche culture discursive du radicalisme anglais des années 1790, dont faisaient aussi partie les Arlequins, les pièces de théâtre, les pièces de monnaie ou encore les caricatures. David Bindman est parvenu à exposer une partie de cet ensemble qu'il a replacé dans le contexte plus vaste des réponses britanniques à la Révolution française ${ }^{44}$. Dans la sélection d'objets qu'il présenta dans The Shadow of the Guillotine, une place particulière est donnée aux représentations visuelles qui évoquent la suppression judiciaire du radicalisme. Ceci n'a rien de surprenant, étant donné la tendance anti-radicale de la décennie 1790. The Prison Diary of John Horne Tooke donne une vision personnelle de la sévérité du quotidien en prison, pour un réformateur qui attendait d'être jugé en $1794{ }^{45}$. Si les fruits et les légumes que lui apportait régulièrement sa fille adoucissaient les effets de la détention, il éprouva des moments plus pénibles quand, pour un temps, deux gardiens 
séjournèrent en permanence dans son cachot. En dépit de l'aspect déresponsabilisant de l'emprisonnement, une anthologie récente de la littérature de prison des radicaux montre qu'une forme puissante de contre-culture émergea des cénacles réformateurs de la prison de Newgate ${ }^{46}$.

Cette anthologie contient aussi des extraits du journal de Charles Pigott, The Jockey Club [qui paraît en 1792]. Pigott était un satiriste radical flamboyant qui mourut prématurément en 1794 d'une fièvre qu'il avait probablement attrapée en prison, alors qu'il attendait d'être jugé pour sédition en 1793. Avant de mourir, Pigott avait achevé son Political Dictionary, qui fut publié de façon posthume et vient d'être réédité dans une version moderne ${ }^{47}$. Ce texte, on ne peut plus fascinant, est un exercice de transgression linguistique, et constitue une réponse radicale à la rhétorique étatique de domination. Comme tel, le dictionnaire de Pigott faisait partie de la bataille qui se déroulait dans les années 1790 autour du contrôle du langage politique. Ce combat fut mené à la fois en public et en privé, non seulement par les radicaux «durs », comme Pigott, mais aussi par les réformateurs qui fréquentaient les sphères policées du radicalisme.

Les discours des réformateurs de la bonne société ont sans doute moins focalisé l'attention des éditeurs universitaires que ceux des radicaux plus populaires. Youth and Revolution révèle la sincérité et l'intensité du radicalisme de certaines élites, à travers la correspondance de jeunes avocats comme William Pattisson, Thomas Amyot and Henry Crabb Robinson ${ }^{48}$. La recherche a aussi progressé de façon significative dans la sauvegarde des écrits des intellectuels radicaux de la fin du XVIII ${ }^{e}$ siècle. Le sermon de Richard Price qui motiva l'écriture des Réflexions de Burke a été deux fois réédité dans des éditions modernes ${ }^{49}$ et sa correspondance, qui aborde des sujets comme la religion, la théologie, la politique et la liberté, a été rassemblée dans un recueil définitif par W. Bernard Peach et D.O. Thomas ${ }^{50}$. La réédition des œuvres de William Godwin et de Mary Wollstonecraft est l'un des meilleurs indicateurs de la vitalité ininterrompue et de l'ampleur du travail éditorial sur les sources du radicalisme des années 1790. Ces deux auteurs, Godwin "l'anarchiste philosophe », et Wollstonecraft la féministe radicale, étaient des penseurs très influents de leur temps. Il existe aujourd'hui pas moins de cinq éditions des Memoirs of the Author of the Vindication of the Rights of Woman de W. Godwin ${ }^{51}$, tandis que les écrits politiques les plus influents de Godwin, en particulier Political Justice (1793), et de Wollstonecraft, notamment A Vindication of the Rights of Woman (1792), sont édités dans deux recueils qui font autorité ${ }^{52}$.

\section{Les études générales et régionales sur le radicalisme}

18 L'intérêt éditorial pour la reproduction des textes radicaux des années 1790 explique aussi le succès des monographies sur le radicalisme anglais. Lorsque Philip Brown et G.S. Veitch ${ }^{53}$ ont les premiers au XX $\mathrm{XX}^{\mathrm{e}}$ siècle étudié le radicalisme anglais, ils insistaient en général sur la force du mouvement de réforme. Cette perspective a atteint un nouvel apogée dans les années 1960 avec le livre séminal d'E.P. Thompson, La formation de la classe ouvrière anglaise ${ }^{54}$, et à un moindre degré, avec les ouvrages de Carl B. Cone et Gwyn A. Williams ${ }^{55}$. Ces travaux faisaient des années 1790 l'ère d'une prise de conscience collective et sans complexe chez les artisans et les ouvriers, tandis que ces années voyaient aussi l'émergence d'une confiance politique ascendante. L'animation puis la responsabilisation du prolétariat, pour reprendre un anachronisme marxiste, étaient interprétés comme une conséquence première de la Révolution française en 
Angleterre. Alors que la marée du marxisme britannique commença à refluer dans les années 1960 et 1970, cependant, des jugements plus mesurés sur l'influence politique de la Révolution française firent surface. En 1979, le livre d'Albert Goodwin, The Friends of Liberty, était la première étude globale du mouvement de réforme démocratique anglais de la fin du XVIII siècle qui donnait une substance au loyalisme; mais cette discussion n'était pas centrale et était subsumée dans le grand récit du mouvement réformateur ${ }^{56}$. À l'autre extrémité idéologique, on trouve les travaux de J.C.D. Clark, qui fait du radicalisme de cette période un mouvement marginal et sans importance, tout du moins par rapport aux discours loyalistes ${ }^{57}$.

19 Les analyses les plus convaincantes du radicalisme des années 1790 (ou, pour être plus exact, des réponses politiques à la Révolution française) sont parvenues à concilier la perspective marxiste d'E.P. Thompson et le «conservatisme dédaigneux» de J.C.D. Clark. Les jugements tempérés de Harry Dickinson occupent ici une position saillante. Dans son British Radicalism and the French Revolution 1789-1815 et The Politics of the People in Eighteenth-Century Britain, cet historien reconnait l'importance et les succès du radicalisme populaire, tout en soulignant les succès écrasants du loyalisme ${ }^{58}$. C'est une perspective similaire qui est adoptée par Jennifer Mori et par Michael J. Turner ${ }^{59}$.

Cependant, les travaux du second, contrairement au livre de la première, s'achèvent sur le triomphe partiel du camp réformateur au plan constitutionnel. Une telle trajectoire chronologique, qui métamorphose la « défaite » radicale des années 1790 en la victoire réformatrice des années 1830 , risque d'estomper la nature intrinsèque du radicalisme anglais et le contexte spécifique de la décennie qui suit la Révolution française. Le recueil d'articles édité par Colin Jones sur Britain and Revolutionary France donne une épaisseur à ce contexte, en évoquant par exemple la " guerre » secrète mal coordonnée par les Français opérant en Angleterre confrontée à la campagne anglaise de propagande contre-révolutionnaire efficacement menée ${ }^{60}$. Ce type de comparaison rend d'autant plus pertinent le traitement parallèle par H.T. Dickinson des conspirations radicales et des succès loyalistes dans les années 1790.

Pourtant, on notera sans réelle surprise que les études générales sur le radicalisme ont tendance à accentuer les succès, si ce n'est la force, de ce mouvement. Ainsi, dans le livre de J. Ann Hone ${ }^{61}$, For the Cause of Truth, le mouvement radical londonien renaît de ses cendres tel un phénix, dans le sillage de l'adoption des Gagging Acts de 1795. La même remarque vaut pour le début des années 1790, pour lesquelles Ray Hemmings peint le tableau d'un mouvement démocratique revigoré et rebelle, à travers les vies de Thomas Hardy et John Cartwright ${ }^{62}$. Dans les deux cas, le loyalisme est vu moins comme un discours dominant que comme une sorte de croyance injuste et réactionnaire, propre à une part importante de la société anglaise, qui reste pourtant par certains aspects impossible à définir.

La démonstration la plus vigoureuse et certainement la plus longue en faveur de la force du radicalisme populaire des années 1790 est l'étude en deux volumes de Jenny Graham, The Nation, the Law and the King ${ }^{63}$. Dans cette enquête capitale sur la politique réformatrice, cette historienne suggère que les idées radicales étaient suffisamment répandues et suffisamment révolutionnaire, pour préoccuper et inquiéter l'élite gouvernante et les autorités. Le livre ne tombe pas dans la dissection thompsonienne de la notion de classe et du radicalisme, dans laquelle la classe ouvrière est vue comme une entité autonome : il suggère au contraire une association bien plus étroite entre les réformateurs artisans et ceux des classes moyennes. L'ouvrage de J. Graham est donc 
un tournant dans la recherche empirique, ne serait-ce que par son ampleur: une monographie en deux volumes de plus de 1000 pages. Malgré son étendue et la richesse $\mathrm{du}$ récit, ce travail ne parvient pas à fournir une interprétation culturelle globale $\mathrm{du}$ mouvement radical des années 1790 : c'est davantage un exposé chronologique de ce qui s'est passé.

En fait, le livre de J. Graham échoue à restituer de façon un tant soit peu approfondie les connexions régionales, les développements et les progrès du radicalisme anglais, dont A. Goodwin avait tracé les contours dans The Friends of Liberty ${ }^{64}$. Par beaucoup d'aspects, ceci est révélateur d'un désintérêt plus général de l'historiographie pour l'activité réformatrice à l'échelle locale, qui a été négligée au profit d'un intérêt pour les mouvements plus vastes. Deux études constituent de notables exceptions. Edwin Jaggard analyse un siècle de politique en Cornouailles à partir de 1790, testant des modèles de comportement électoral afin de montrer la montée en puissance progressive du libéralisme non-conformiste ${ }^{65}$. Dans le livre de John Stevenson, qui est centré sur Sheffield, le renforcement du radicalisme émerge bien plus tôt : un milieu réformateur dynamique s'y enracine au début des années 1790. C'est là aussi, pratiquement, que la première organisation politique de la classe ouvrière fut fondée en Grande-Bretagne, sous les auspices de la Sheffield Society for Constitutional Information ${ }^{66}$.

\section{Les liens du radicalisme anglais avec le continent}

De même que les ramifications provinciales du radicalisme, ses liens avec le continent n'ont fait l'objet que de très peu d'études. À de nombreux égards, ceci est le fruit du contexte historique. Contrairement à ce que pourraient laisser penser les décrets de fraternité annoncés par les Français au début des années 1790 et les croyances alarmistes de certains contemporains, aucune cellule radicale anglaise d'importance ou de durée de vie conséquente n'opéra en France. Celle qui se rapproche le plus d'une telle description est le British Club à Paris, fondé en 1792. Le livre splendide de David V. Erdman sur John Oswald, soldat et républicain activiste a permis de faire la lumière sur cette organisation ${ }^{67}$. Oswald est né à Edinburgh, mais dès le milieu des années 1780 il vivait et travaillait à Londres, comme journaliste et éditeur, en compagnie de James Ridgway. Avant de quitter l'Angleterre pour Paris en 1790, il se lia d'amitié avec des sommités démocrates comme Thomas Paine, James Mackintosh et John Horne Tooke. À Paris, il fréquenta des hommes comme les expatriés radicaux Thomas Cooper, Joel Barlow, Thomas Christie, Wolfe Tone et Henry Redhead Yorke. Oswald se vit octroyer la citoyenneté française honoraire et devint commandant dans l'armée française l'année même où le British Club fut créé, mais il mourut lors des guerres de Vendée en septembre 1793.

Tandis que les relations qu'entretenait Oswald avec la France étaient volontaires, Protest and Punishment de George Rudé retrace l'histoire collective du lien forcé entre les réformateurs anglais déportés et l'Australie ${ }^{68}$. Le récit ne se limite pas à l'Angleterre, mais englobe aussi la Grande-Bretagne, l'Irlande et le Canada, de la fin du XVIII ${ }^{e}$ siècle au milieu du siècle suivant. En tant que tels, les déportés des années 1790, en particulier anglais, ne sont évoqués qu'en passant. Cependant, cette analyse des détenus politiques les distingue des criminels de droit commun avec lesquels ils étaient transportés, et démontre qu'ils étaient, pour la plupart, traités en «Britanniques libres " (freeborn 
Britons) dans la colonie. À part l'exception rare du bandit, Maurice Margarot, qui fut déporté à Botany Bay en 1794 en compagnie des "martyrs » écossais, la plupart des réformateurs exilés des années 1790 abandonnèrent leurs activités politiques et vécurent dans une retraite paisible.

Ce ne fut pas le cas de ceux qu'on pourrait nommer les jacobins anglo-américains. À la lecture du travail de Richard J. Twomey, il semble que les radicaux anglais exilés en Amérique, de même que leurs homologues irlandais et écossais, se transformèrent presque toujours en journalistes jeffersoniens influents et en leaders d'organisations politiques locales ${ }^{69}$. Mais la vaste majorité des exilés radicaux outre-Atlantique se retirèrent complètement de la vie publique. R. J. Twomey, ainsi d'ailleurs que Michael Durey ${ }^{70}$, s'intéressent aux réseaux qu'établirent la minorité des radicaux anglais dans la jeune république américaine. Ces deux historiens choisissent une approche biographique pour dresser le portrait démographique et décrire les activités des réfugiés radicaux. Ils montrent ainsi que ces derniers, qui détenaient pour beaucoup un savoir-faire en matière de publications d'ouvrages politiques et de pamphlets, avaient une position centrale pour influencer, si ce n'est transformer, le discours démocratique de la république. Ils ne constituaient pourtant pas un groupe unitaire, mais plutôt une collectivité disparate et souvent divisée, avant tout unie par les idées radicales de liberté et d'égalité importées de leur pays natal.

\section{Biographies radicales}

L'approche prosopographique de G. Rudé, R. Twomey et M. Durey permet de comprendre les expériences partagées par les radicaux en exil. Pour avoir une analyse plus détaillée des vies de réformateurs spécifiques, il faut se tourner vers les biographies individuelles. La majorité des efforts qui ont été consacrés à retracer la vie des réformateurs du XVIII ${ }^{e}$ siècle se sont focalisés sur l'intelligentsia radicale. David Williams, par exemple, a fait l'objet d'une biographie convaincante : ce théoricien du droit et de la politique, né au Pays de Galles et fondateur du Literary Fund, affirmait que ses Letters on Political Liberty (1782) avaient influencé la pensée des révolutionnaires français ${ }^{71}$. Il se vit accorder la citoyenneté française en 1792, en compagnie de T. Paine, J. Mackintosh, R. Price, G. Washington et J. Priestley. Ce dernier, dont les œuvres vont de la découverte de l'oxygène à des recherches en physique, philosophie, théologie, éducation et politique, attire naturellement le biographe ${ }^{72}$. La vie de Priestley en Angleterre fut abruptement interrompue lorsque son réformisme inspira les Priestley Riots (" émeutes Priestley ») en 1791, l'obligeant finalement à s'exiler en Amérique. Le livre de Jenny Graham offre une bonne vue d'ensemble sur la période américaine de Priestley ${ }^{73}$, tandis qu'un recueil d'articles récent s'intéresse à sa carrière manquée à Birmingham ${ }^{74}$. Toutefois, malgré ces études, Priestley donne parfois l'impression d'être un intellectuel agité, qui inspira autant de controverses que d'innovations. Dans la biographie définitive de Robert E. Schofield, pourtant, il est présenté comme un scientifique empiriste total, qui possédait d'excellentes bases en métaphysique, en politique et en philosophie naturelle, tout en ayant des opinions bien tranchées sur la liberté religieuse et politique, ce qui faisait de lui une figure centrale des Lumières anglaises ${ }^{75}$.

William Godwin, le philosophe-anarchiste, est une autre figure-clef des Lumières qui a reçu une attention particulière des chercheurs. C'était l'un des intellectuels radicaux 
les plus hautement considérés dans les années 1790, dont l'œuvre politique et fictionnelle fut prolifique. Cependant, lorsque Kegan Paul écrivit la première biographie de Godwin en 1876, il estimait que « la génération actuelle n'a que peu lu ses œuvres $"{ }^{76}$, point de vue qui a été plus récemment exprimé par Ian Ward, qui voit en Godwin " une personnalité quelque peu oubliée " ${ }^{77}$. Cependant, une telle conclusion fait très peu de cas de l'historiographie récente sur Godwin. On a déjà mentionné la richesse des sources rééditées sur Godwin, tandis que son pamphlet le plus célèbre a bénéficié d'un examen minutieux dans un livre de Mark Philp ${ }^{78}$. En outre, sa vie et sa carrière sont aujourd'hui reconnues à leur juste valeur dans les biographies de Don Locke et Peter Marshall ${ }^{79}$. Ces deux livres situent l'originalité de sa pensée politique et morale, ainsi que son talent de romancier, dans le contexte du monde social et politique dans lequel il évoluait, et par rapport à sa vie personnelle.

D'aucuns se souviennent de Godwin avant tout comme du mari de Mary Wollstonecraft et père de Mary Shelley: une biographie de la famille par William St Clair ne fait que renforcer cette identification ${ }^{80}$. Alors que le féminisme radical génère énormément d'intérêt idéologique et historique de la part des chercheurs, c'est fort logiquement que M. Wollstonecraft a suscité à son tour l'attention des biographes. Les travaux de Gary Kelly, Janet Todd, Diane Jacobs, Claudia L. Johnson, Barbara Taylor, et plus récemment encore, Lyndal Gordon, font l'articulation entre la pensée pionnière et la vie fascinante de $\mathrm{M}$. Wollstonecraft ${ }^{81}$. Chacun de ces livres souligne la place de cette dernière dans l'histoire du féminisme et compatit avec ses défauts émotionnels et ses dilemmes sexuels, qui sont le fruit de la domination masculine, dans une société où les femmes étaient marginalisées. Le prétexte qui motive la biographie de D. Jacobs, publiée peu de temps après l'étude déjà classique de $\mathrm{J}$. Todd, est la découverte de lettres qu'avait cachées l'éditeur de $\mathrm{M}$. Wollstonecraft, Joseph Johnson. On traverse ici la frontière entre le monde policé de l'intellectuelle radicale et le monde du radicalisme anglais, qui s'appuyait sur les médias. Ici, les études de la période romantique rencontrent les analyses de la politique populaire. Une étude récente de la carrière de J. Johnson révèle l'enchevêtrement de ses rôles (auteur, éditeur, imprimeur et libraire), et la nette alliance entre ses activités de publication et la mise en avant de ses thèses radicales, tant au plan politique que religieux ${ }^{82}$.

J. Johnson était en fait un lien commun à de nombreux libres penseurs et auteurs radicaux des années 1790. Il publia Paine, dont le parcours et l'existence nous ont été racontés par Jack Fruchtman, John Keane et Trevor Griffiths ${ }^{83}$. La biographie est un récit construit, et le risque de dériver vers l'hagiographie n'est jamais très loin, en particulier avec un personnage comme Paine. Pourtant, chacun des livres mentionnés admet les faiblesses de Paine et cherche à expliquer ses contradictions apparentes. Paine n'apparait jamais comme un héros parfait et tous ces ouvrages se lisent avec plaisir. Le meilleur est de loin celui de J. Fruchtman, tandis que la biographie de T. Griffiths est trop courte et celle de J. Keane contient trop d'erreurs pour rivaliser avec le premier cité ${ }^{84}$.

31 Parmi les auteurs publiés par J. Johnson, Paine était l'un des plus saillants au début des années 1790 , reliant les journalistes et l'intelligentsia radicale; au milieu des années 1790 , c'étaient en revanche les jeunes poètes romantiques William Wordsworth et Samuel Taylor Coleridge qui attachèrent de façon plus étroite le monde du radicalisme policé et celui du radicalisme plébéien. Si l'idée, avancée par Kenneth Johnson, d'un Wordsworth espion à la solde du gouvernement, et fréquentant les cercles radicaux en 
tant qu'agent secret et contre-révolutionnaire, a été réduite à néant ${ }^{85}$, on sait en revanche que Wordsworth et Coleridge, ainsi que Robert Southey, entretenaient des contacts étroits avec les jacobins londoniens entre 1794 et $1795^{86}$. De façon identique, Nicholas Roe a produit une synthèse biographique sur Wordsworth et Coleridge après 1790, qui situe leurs activités et leurs écrits dans le contexte plus large du radicalisme, et dévoile la complexité du milieu dans lequel ils vivaient ${ }^{87}$. Dépassant la biographie historique classique, ce dernier livre propose une analyse de la culture politique radicale qui est modelée et même façonnée par le radicalisme littéraire de la période. Il montre comment d'un côté, les vies intellectuelles et politiques de poètes romantiques qui s'enthousiasmaient alors pour la réforme, et de l'autre le radicalisme populaire, ne coexistaient pas seulement, mais bien souvent se chevauchaient.

C'est là que se situent les perspectives les plus riches de renouvellement biographique sur les radicaux, en partant de cette strate de la réforme populaire. En dehors d'une vie de John Horne Tooke ${ }^{88}$, et d'un essai biographique du caricaturiste radical, Richard Newton ${ }^{89}$, il n'existe pas d'étude significative sur la vie des leaders démocratiques de l'époque. Cette lacune est souvent due au manque de sources manuscrites, mais ceci ne saurait suffire à expliquer pourquoi, par exemple, le fondateur de la LCS, Thomas Hardy, n'a suscité l'intérêt d'aucun biographe, ou pourquoi les historiens contemporains ne se sont pas intéressés au principal orateur jacobin des années 1790, John Thelwall. La description et la discussion de telles destinées livreraient de précieux éclaircissements sur les protagonistes du radicalisme anglais.

\section{L'idéologie radicale}

33 À la différence des biographies, qui dans la majorité des cas ne déclenchent pas beaucoup de débats interprétatifs, l'idéologie est un domaine plus litigieux. Philip Harling a remarqué qu'» en ce moment il n'y a ni une école interprétative dominante, ni deux ou plus qui s'affrontent " parmi les historiens de la politique britannique de la fin du XVIII et du début du XIX siècle : «On est libre de suivre ses pensées là où elles mènent sans peur réelle d'être massacré à l'écrit ou enfermé dans un rôle " ${ }^{90}$. Le seul domaine dans lequel le désaccord a émergé, à un certain degré, est l'historiographie de l'idéologie radicale. Comme nous l'avons vu, la Révolution française est à l'origine d'une guerre idéologique d'une ampleur sans précédent en Angleterre, une guerre qui polarisa l'opinion politique entre loyalistes et radicaux. Les historiens qui ont étudié la dimension philosophique et politique de l'idéologie radicale ont eu tendance à mettre le républicanisme au cœur du questionnaire ${ }^{91}$. Une école de pensée a ainsi considéré que l'idéologie radicale anglaise des années 1790 se référait à une conception rétrograde des droits historiques. Des historiens comme Bernard Bailyn, Herbert Butterfield et Ian Christie ont soutenu l'idée que cette idéologie radicale tenait à restaurer les droits politiques des anciens Anglo-Saxons ${ }^{92}$. Carlo Hay a écrit que «le but fondamental des radicaux anglais [était] [...] de restaurer de mythiques années passées ${ }^{93}$. Le principal tenant de ce courant, dans l'historiographie récente, est J.G.A. Pocock, qui voit dans les «vertus républicaines classiques » la tradition dominante de l'idéologie radicale anglaise ${ }^{94}$.

Cette approche contraste avec une perspective plus traditionnelle, qui considère que l'idéologie radicale anglaise de la fin du XVIII ${ }^{e}$ siècle était moins fascinée par l'historicisme nostalgique identifié par J.G.A. Pocock et les autres, que par la 
revendication des droits naturels. Le défenseur le plus convaincant de ce modèle théorique, dans les années récentes, est Isaac Kramnick ${ }^{95}$. D'après lui, l'interprétation de Pocock «est fondamentalement fallacieuse », car «ce qui donne sa cohérence au radicalisme anglais du XVIII ${ }^{e}$ siècle est son désir même "de détruire l'ordre existant et de créer de nouveau". Le radicalisme anglais voulait renverser l'ordre social du rang et du privilège, la société statique et stratifiée de la détermination " ${ }^{96}$. Comme Harry Dickinson l'a noté : "Il semble assez clair [...] que les idées lockiennes des droits naturels et du contrat originel firent un retour spectaculaire et décisif chez les radicaux de la fin du XVIII ${ }^{e}$ siècle " ${ }^{97}$. Cette orientation théorique apparut le plus clairement dans les écrits des propagandistes politiques des classes populaires. Gregory Claeys a explicité l'idéologie politique de Paine par rapport au contexte d'un droit naturel et d'une pensée des droits qui émergent au XVIII e siècle, faisant de l'auteur des Droits de l'homme le principal protagoniste du développement du langage des droits naturels ${ }^{98}$. John Thelwall était un autre radical anglais des années 1790 dont l'idéologie était enracinée dans la théorie des droits naturels, et ceci est montré de façon convaincante dans les Seditious Allegories de Michael Scrivener, qui est fondamentalement une étude des écrits politiques, oratoires et journalistiques de Thelwall, autant que de son discours littéraire ${ }^{99}$.

Si ces études mettent l'accent sur le rôle de la philosophie politique dans le débat idéologique des années 1790, un autre courant récent a montré que la religion fut également centrale dans la formation du radicalisme à la fin du XVIII ${ }^{e}$ siècle ${ }^{100}$. Certaines études ont ainsi relevé que l'agitation politique était ancrée dans le discours radical chrétien, qui parlait le langage des droits par rapport aux dons divins de création, de bienveillance pour les pauvres, et des idées de libération des peuples opprimés présentes dans les Gospels ${ }^{101}$. En outre, la pensée politique et philosophique de la fin du XVIII ${ }^{e}$ siècle est inextricablement liée aux langages de la dissidence religieuse. Ce domaine est brillamment exploré par Stuart Andrews, qui met au jour les critiques unitariennes contre les institutions, à travers les sermons doctrinaux, les pamphlets et les débats parlementaires ${ }^{102}$.

\section{La culture du radicalisme}

Le livre de S. Andrews indique les liens entre l'idéologie radicale et l'activisme d'opposition. D'une façon bien plus ambitieuse, le Liberty and Property d'Harry Dickinson y parvient aussi. Il s'efforce, comme l'écrit l'auteur, « de rattacher les idées politiques à l'action politique, d'articuler ce que les hommes écrivaient et disaient sur la nature humaine, sur la société civile et sur le gouvernement civil, avec la compréhension de ce qu'ils firent en réalité » ${ }^{103}$. Ce type de démarche, tournée vers l'étude des pratiques concrètes de la politique et l'analyse de la culture du radicalisme, a engendré une historiographie particulièrement innovante sur la politique populaire anglaise dans les années récentes ${ }^{104}$. Elle a bénéficié de l'émergence et de l'influence des cultural studies postmodernes, qui ont déplacé le champ au-delà du matérialisme et de l'empirisme marxiste de l'école d'E.P. Thompson. Un des premiers travaux majeurs à engager la discussion avec Thompson et à contester sa conception du radicalisme populaire des années 1790, fut l'œuvre de Craig Calhoun en $1982{ }^{105}$. Ce dernier développe une analyse conceptuelle et historique subtile, qui interroge le radicalisme sous l'angle de ses 
rituels sociaux et de sa culture symbolique, en le replaçant dans son contexte idéologique et de conscience de classe.

Si C. Calhoun a mis en lumière la continuité de l'activité culturelle radicale entre la fin du XVIII ${ }^{e}$ siècle et le début du XIX ${ }^{e}$ siècle, Iain Mc Calman est devenu le chef de file de cette argumentation. Son Radical Underworld est un point de repère incontournable pour toute étude du mouvement démocratique, établissant un lien étroit entre le jacobinisme londonien et le monde émergeant des socialistes agrariens du XIX ${ }^{e}$ siècle ${ }^{106}$. Iain Mc Calman maîtrise quantité de sources archivistiques au profit d'un exposé fascinant et bien écrit de la sous-culture du radicalisme. Son étude reconstitue la richesse du milieu culturel des ultra-radicaux, permettant au lecteur d'imaginer le fonctionnement et les intrigues des pubs, clubs, tribunes, bordels et théâtres qui composaient les espaces de la politique radicale anglaise. Il est important de noter que ce type de géographie historique, situant la politique populaire dans un paysage culturel, dépasse la tradition historiographique des études marxistes britanniques, qui donne sa marque au concept de sphère publique radicale.

Par de nombreux aspects, on peut considérer que le livre de I. Mc Calman a nourri l'inspiration d'un ensemble de travaux regroupés sous le nom d'» études plébéiennes » ${ }^{107}$ en particulier ceux de David Worrall et de Jon Mee ${ }^{108}$. Dans ces deux ouvrages, la culture radicale est présentée à travers divers discours d'opposition, dans lesquels les formes de domination hégémonique étaient contestées et manipulées. Ce concept de politique spatiale a gagné en sophistication sur le plan théorique depuis la traduction anglaise de l'ouvrage d'Habermas sur l'opinion publique, qui délimite les contours d'une sphère publique bourgeoise se formant dans des arènes comme la presse et le café. Tandis que le processus historique, tel que présenté par Habermas, exclut spécifiquement la sphère publique, des révisionnistes comme Geoff Eley, Terry Eagleton et Jon Klancher ont donné sens au concept d'une contre-sphère publique du radicalisme, populaire et puissante ${ }^{109}$. Kevin Gilmartin a donné sens à ce concept, en étudiant dans Print Politics le langage et l'activité des radicaux tels qu'ils étaient véhiculés dans la presse du début du XIX ${ }^{e}$ siècle ${ }^{110}$. Ce livre souligne comment des publicistes radicaux, tels que Thomas Wooler et Richard Carlile, étaient au centre du discours radical, comment la presse véhicula le changement culturel et politique, et comment elle "pouvait fournir l'instrument de transition par lequel le peuple réclamait l'autorité dans la Chambre des Communes, qui leur avait été retirée par la corruption ${ }^{111}$.

L'étude de Stuart Andrews sur les périodiques de l'époque donne une importance similaire à la culture de l'imprimé dans les années $1790{ }^{112}$, tandis que le travail de Paul Keen décrit les textes des littérateurs radicaux comme "un lieu de contestation idéologique " ${ }^{113}$. Par delà cette culture textuelle, le radicalisme a trouvé à s'exprimer dans une grande variété de genres, dont certains sont précisés dans un recueil édité par Robert Maniquis ${ }^{114}$. En outre, Marcus Wood propose une analyse élaborée de la manière dont des journalistes des années 1790 comme Daniel Isaac Eaton et Thomas Spence recouraient à la satire populaire pour exprimer une critique radicale. Il montre aussi que ce type de critique n'était pas limité aux pamphlets et au monde de l'imprimé, mais se transmettait à travers une variété de médias ${ }^{115}$. Le discours radical anglais trouvait un débouché dans tout un tas d'objets imprimés et de manifestations déjà évoqués. Concernant les caricatures, elles ont été brillamment analysées par H. T. Dickinson et, plus récemment, par Tamara L. Hunt ${ }^{116}$. 

est au centre des travaux de James Epstein ${ }^{117}$. Chez les réformateurs de la fin du XVUI siècle, le radicalisme s'exprime dans les représentations et la pratique du discours, $d u$ rituel et du symbolisme. À partir de ces constructions culturelles, J. Epstein explore les lieux de la politique populaire. Il articule son récit du radicalisme avec une réflexion passionnante sur la performance et sa relation avec différents espaces discursifs ${ }^{118}$. Du café à la salle d'audience, c'est toute la culture radicale des années 1790 qui est réinterprétée, ce qui permet de voir non seulement comment le radicalisme s'exprimait mais aussi comment il était représenté, au sens théâtral du terme ${ }^{119}$.

\section{La menace de révolution}

41 Tandis que l'étude de la culture radicale est un terrain d'enquête relativement nouveau, notamment influencé par le postmodernisme, un sujet a depuis longtemps intéressé les historiens : la menace révolutionnaire dans les années 1790. Malgré toute l'agitation de la période et l'expression consacrée de l'» ère des révolutions » (E. Hobsbawm), la Grande-Bretagne échappa au type de changements sociaux et politiques qui se produisirent en France. Mais il y eut des signes inquiétants. John Brewer et John Styles ont décrit les Anglais comme un " peuple ingouvernable » au XVIII ${ }^{e}$ siècle, et Ian Gilmour a montré que tout au long du siècle, les manifestations et les émeutes violentes proliférèrent ${ }^{120}$. La dernière décennie du XVIII ${ }^{e}$ siècle vit d'innombrables vagues de révoltes, comme John Bohstedt l'a montré ${ }^{121}$, et l'on connaît la force des foules, en tant qu'entité politique populaire ${ }^{122}$. Si Charles Tilly a suggéré que les émeutes furent progressivement abandonnées au profit des meetings publics, des manifestations et des pétitions au cours du XVIII ${ }^{\mathrm{e}}$ et du XIX ${ }^{\mathrm{e}}$ siècles ${ }^{123}$, il n'en reste pas moins qu'un niveau de violence collective visible et inquiétant subsistait dans les années 1790. Pour beaucoup, la violence était locale et prenait la forme d'émeutes de subsistance, qui étaient la réponse à des privations socio-économiques causées par la guerre contre la France ${ }^{124}$. Toutefois, comme ces révoltes étaient avant tout rurales et inspirées par des questions matérielles, les radicaux-réformistes urbains, qui représentaient la plus grande partie du mouvement favorable à la réforme, échouèrent pour l'essentiel à exploiter ce mécontentement populaire.

En dépit de ces occasions manquées, nombreux furent ceux parmi les contemporains, qui croyaient que les revendications sociales exprimées par les émeutiers, et l'activisme politique grandissant généré par la Révolution française, faisaient de l'Angleterre une pépinière de révolutionnaires. La réalité de la menace révolutionnaire et la manière dont la Révolution fut évitée restent des questions qui suscitent le débat. I. Gilmour, par exemple, reconnaît l'étendue de la protestation populaire, mais considère que l'absence de Révolution en Angleterre fut avant tout due à la modération et à la compétence du gouvernement ${ }^{125}$. I.R. Christie, à l'inverse, nie presque la menace radicale: la modération sociale et politique du peuple britannique et la puissance de l'État rendirent nul et non avenu tout désir d'insurrection ${ }^{126}$. De son côté, Edward Royle pense que la solidité de l'establishment a sapé « tout espoir réaliste d'une révolution victorieuse ${ }^{127}$.

43 En partie, cet espoir aurait pu bénéficier du vaste courant d'anti-monarchisme des années 1790, qui dériva dangereusement à certains moments vers le régicide ${ }^{128}$. Cependant, selon Malcom I. Thomis et Peter Holt, les révolutionnaires ne furent jamais

Annales historiques de la Révolution française, 342 | octobre-décembre 2005 
qu'un groupe marginal qui ne représentait aucune menace réelle pour le statu quo : ils ne s'attirèrent jamais le soutien de masse nécessaire pour convertir leur mécontentement en une révolution véritable ${ }^{129}$. Dans La formation de la classe ouvrière anglaise pourtant, E.P. Thompson insiste sur l'insurrection constitutive de la politique radicale à la fin du XVIII ${ }^{e}$ siècle. Une bonne partie de l'historiographie récente, sans forcément valider l'ensemble de la thèse thompsonienne, a soutenu l'idée qu'une composante révolutionnaire non-négligeable existait au sein des jacobins anglais. Dans sa reconstruction du monde radical, I. McCalman donne ainsi accès au milieu brutal et convivial des insurgés londoniens, dont il démontre l'influence et la pression hautement subversive qu'il exerçait sur le mouvement radical anglais ${ }^{130}$. Cette interprétation est affirmée avec plus de poids encore par Roger Wells ${ }^{131}$.

\section{Répression et poursuites judiciaires}

Malgré le débat historiographique sur l'étendue de la menace révolutionnaire des années 1790, un point reste incontesté: la nervosité qu'engendra la montée du radicalisme dans les cercles gouvernementaux. Lorsque le spectre de la révolution se dessina, une campagne de répression légale fut mise en place, que certains historiens ont appelée un "règne de terreur " ${ }^{132}$. Les procès politiques se multiplièrent, à mesure que le gouvernement essayait de refroidir l'enthousiasme des radicaux, en particulier ceux qui travaillaient dans les métiers du livre ${ }^{133}$. Étonnamment, aucune analyse importante des procès radicaux dans les années 1790 n'a été écrite jusqu'à présent ${ }^{134}$. Les célèbres procès pour trahison de 1794, qui débouchèrent sur l'acquittement de Thomas Hardy, John Horne Tooke et John Thelwall, ont fait l'objet de l'étude sans relief d'Alan Wharam ${ }^{135}$. Ces procès bénéficient d'un traitement bien supérieur dans le livre majestueux de John Barrell ${ }^{136}$, qui explore avec moult détails les arguments politiques, légaux et publics formulés au milieu des années 1790, afin d'évaluer à quel point les radicaux imaginaient la mort de George III. Aux côtés des livres d'E.P. Thompson et de I. McCalman, cette lecture gratifiante est incontournable à tout chercheur ou étudiant intéressé par le radicalisme de la fin du XVIII ${ }^{e}$ siècle. L'étude de John Barrell ne se limite pas aux procès pour trahison de 1794, mais intègre aussi les prophéties de Richard Brothers, la prise d'armes de la LCS, les buts de la conspiration dite des pop-gun de 1794, et les poursuites de radicaux en Écosse.

L'une des conclusions les plus importantes que l'on peut déduire de cette analyse des procès politiques est la manière dont ils fonctionnaient en tant qu'événements discursifs. De fait, la poursuite des radicaux donne une clef d'interprétation de la manière dont s'entrelaçaient les cultures politique et légale dans les années 1790. La loi ne fonctionnait pas comme un pur système abstrait de justice, mais bien comme un «système culturel», faisant usage de types de performances et de discours spécifiques. Pour tester cette notion, la salle d'audience, et les drames qui se nouaient souvent à l'extérieur et après le procès, est un espace parfait. C'était une arène publique où des récits concurrents entraient en jeu, un lieu de contestation où l'opinion publique et les attitudes envers la loi pouvaient être mesurés, et où les idées de justice et de droits politiques n'étaient pas seulement proclamées, mais également débattues et façonnées. Ces thématiques ont été explorées dans les travaux récents de J. Epstein et Michael T. Davis, qui ont montré comment les procès aidèrent à formuler, à légitimer, à articuler et à animer l'action politique populaire ${ }^{137}$. 
De façon similaire, des travaux récents ont montré comment les réformateurs emprisonnés dans la prison de Newgate pendant la Révolution française parvinrent à développer une forme de résistance culturelle depuis l'intérieur de la prison. Un milieu jacobin, en quelque sorte, se forma à Newgate, qui exerçait un attrait sur les réformateurs policés et plébéiens de la période. Lieu contesté de formation, de négociation et de résistance radicales, la prison regonfla les enthousiasmes individuels, mais parvint aussi à consolider le mouvement de réforme dans son ensemble.

Pour conclure, on peut brièvement évoquer les limites de cet article. Il n'était aucunement question de faire une étude exhaustive de ce champ historiographique, mais plutôt de donner une vue d'ensemble sur les principaux livres et articles portant sur le radicalisme des années 1790 publiés durant les trente dernières années. On a donné une apparence de cohérence à cette masse bibliographique, en distinguant quelques-uns des thèmes historiographiques fondamentaux, et en analysant comment des travaux spécifiques ont contribué à notre compréhension de la politique populaire anglaise de la fin du XVIII ${ }^{e}$ siècle. Il est certain que certaines histoires ont été négligées dans cette discussion, et certains thèmes étroitement liés n'ont pu se voir accorder toute l'attention qu'ils méritaient dans l'espace dont nous disposions. Cet article démontre en tout cas clairement la force, la diversité et le caractère innovant des études récentes sur le radicalisme anglais. Des signes importants semblent indiquer que ceci n'est pas prêt de s'arrêter. Le 29 juin 2005, plus de 80 chercheurs bravèrent une chaleur étouffante pour assister à une conférence donnée à l'Institut de Recherche Historique de Londres (IHR) sur «La gauche britannique dans les années 1790 ». Les orateurs insistèrent sur la fertilité du radicalisme anglais pendant la décennie révolutionnaire, et son aptitude à engendrer une rénovation culturelle et politique. Cette conférence a débouché sur le projet de mettre en place un réseau de recherche sur les années 1790 , ce qui confirme que l'étude du radicalisme anglais de cette période est un domaine vivant et épanouissant. Les héritiers de Paine survivent en quelque sorte!

\section{NOTES}

1. Jacques Mallet du PAN, Considérations sur la nature de la Révolution de France, et sur les causes qui en prolongent la durée, Londres et Bruxelles, 1793, p. V.

2. Clive EMSLEY, "The Impact of the French Revolution on British Politics and Society», in Ceri CROSSLEY and Ian SMALL (ed.), The French Revolution and British Culture, Oxford, 1989, p. 33.

3. Voir par exemple Seamus DEANE, The French Revolution and Enlightenment in England, 1789-1832, Cambridge, Mass., 1988; Stephen PRICKETT, England and the French Revolution, Basingstoke, 1989 ; H.T. DICKINSON, «The Impact of the French Revolution on Britain», Contemporary Review, 255, 1989, pp. 20-26.

4. "In England, even more than in France, the history of the French Revolution was being written before the story of it was completed». Hedva BEN-ISRAEL, English Historians on the French Revolution, Cambridge, 1968, p. 3. 
5. Charles James Fox to Richard Fitzpatrick, 30 juillet 1789, in Memorials and Correspondence or Charles James Fox, ed. John RUSSELL, 4 vols., Londres, 1853, II, p. 361.

6. Jacques Pierre Brissot de Warville, 31 décembre 1790, cité in Georges LEFEBVRE, The French Revolution, 2 vols., Londres et New York, 1962, I, p. 217.

7. Conor CRUISE O'BRIEN, «Nationalism and the French Revolution», in Geoffrey BEST (ed.), The Permanent Revolution: The French Revolution and its Legacy 1789-1989, Londres, 1988, pp. 36-37.

8. F.D. CARTWRIGHT (ed.), The Life and Correspondence of Major Cartwright, Londres, 1826, p. 182.

9. H.T. DICKINSON, «Britain and the Ideological Crusade against the French Revolution» in L. DOMERGUE et G. LAMOINE (ed.), Après 89. La Révolution Modèle ou Repoussoir, Toulouse, 1992, p. 155.

10. J.G.A. Рососк, «Political Thought in the English-Speaking Atlantic, 1760-1790. Part 2: Empire, Revolution and the End of Early Modernity», in The Varieties of British Political Thought, 1500-1800, Cambridge, 1993, p. 308.

11. Milan KUNDERA, L'insoutenable légèreté de l'être, Paris, Gallimard, 1984 (trad. François Kérel), p. 323.

12. George RUDÉ, Revolutionary Europe, 1783-1815, Londres, 1964, p. 180.

13. N.C. PHILLIPS, «Burke and Paine: The Conservative and Radical Minds», Landfall, 8 (1954), p. 36.

14. Voir par exemple : Robert R. DOZIER, For King, Constitution and Country: The English Loyalists and the French Revolution, Lexington, 1983 ; J.C.D. CLARK, English Society 1688-1832: Ideology, Social Structure and Political Practice during the Ancien Régime, Cambridge, 1985 ; H.T. DICKINSON, «Popular Conservatism and Militant Loyalism 1789-1815», in H.T. DICKINSON (ed.), Britain and the French Revolution 1789-1815, Basingstoke, 1989, pp. 104-25; Mark PHILP, «Vulgar Conservatism, 1792-93», English Historical Review, 110 (1995), pp. 42-69.

15. M.O. GRENBY, The Anti-Jacobin Novel: British Conservatism and the French Revolution, Cambridge, 2001, p. 4.

16. Cet article sera avant tout centré sur les principaux livres et articles publiés sur le radicalisme anglais lors des trente dernières années.

17. Philip Francis à Edmund Burke, 19 février 1790, in T.W. COPELAND (ed.), The Correspondence of Edmund Burke, 10 vols., Cambridge, 1958-70, VI, p. 86.

18. Pour un survol de cette littérature, voir Gayle TRUSDEL PENDLETON, «Towards a Bibliography of the Reflections and Rights of Man Controversy», Bulletin of Research in the Humanities, 85 (1982), pp. 65-103; Mark PHILP, «Burke and Paine: texts in Context», Enlightenment and Dissent, 9 (1990), pp. 93-105.

19. Thomas COPELAND, Our Eminent Friend Edmund Burke, New Haven, 1949, p. 148.

20. Alfred совBAN (ed.), The Debate on the French Revolution 1789-1800, Londres, 1950, p. 31.

21. Pour une discussion de la chronologie des réponses à Burke, voir : James T. BOULToN, The Language of Politics in the Age of Wilkes and Burke, Londres et Toronto, 1963, pp. 265-271; R.R. FENNESsy, Burke, Paine and the Rights of Man: A Difference of Political Opinion, The Hague, 1963, pp. 181-212.

22. Marilyn BUTLER (ed.), Burke, Paine, Godwin, and the Revolution Controversy, Cambridge, 1984.

23. Gregory CLAEYs (ed.), The French Revolution Debate in Britain: The Origins of Modern Politics, Basingstoke, 2006.

24. Iain HAMPSHER-MONK (ed.), The Impact of the French Revolution: Texts from Britain in the 1790s, Cambridge, 2005.

25. Gregory CLAEYS (ed.), Political Writings of the 1790s, 8 vols., Londres, 1995.

26. Horst IHDE, «The Heirs to Paine's Democratic Tradition», in P.M. KEMP-ASHRAF (ed.), Life and Literature of the Working Class: Essays in Honour of William Gallacher, Berlin, 1966, p. 93. Sur la réception britannique des Droits de l'Homme voir Edward ROYLE, «The Reception of Paine», Bulletin of the Society for the Study of Labour History, 52 (1987), pp. 14-20. 
27. Rights of Man a été publié chez Penguin Classics Series, édité par Eric FONER, Harmondsworth, 198; dans une version éditée par by Gregory CLAEYS, Indianapolis, 1992; et chez Dover Publications, ed. Michael Foot, Mineola, New York, 2000.

28. Thomas Paine Reader, ed. Michael FooT et Isaac KRAMNICK, Harmsondsworth, 1987; Thomas Paine: Political Writings, ed. Bruce KUKLICK, Cambridge, 1989; Thomas Paine: Rights of Man, Common Sense and Other Political Writings, ed. Mark PHILP, Oxford, 1998; et Common Sense, Rights of Man, and Other Essential Writings of Thomas Paine, ed. Jack FRUCHTMAN et Sidney ноOк, New York, 2003.

29. Une édition de The Age of Reason, édité par Philip S. FONER et pour la première fois publié en 1948, a été ressortie chez Citadel Press, New York, 1991. Une édition est aussi disponible chez Dover Publications, par Moncure Daniel ConwAY, Mineola, New York, 2004.

30. An Appreciation of Thomas Paine [CD-ROM]: Bank of Wisdom [1998]. Les principaux travaux inclus sur ce CD-ROM sont : Life and Writings of Thomas Paine, ed. Daniel Edwin WHEELER, 10 vols., New York, 1908; The Writings of Thomas Paine, ed. Moncure Daniel conWAY, 4 vols., New York, 1894; The Life of Thomas Paine, ed. Moncure Daniel ConwAY, 2 vols., New York, 1892; The Life of Thomas Paine, ed. Peter Eckler, New York, 1893; John E. REMSBURG, Thomas Paine, the Apostle of Religious and Political Liberty, Boston, 1880; and G. VALE, The Life of Thomas Paine, New York, 1840.

31. The State Trials on CD-ROM [CD-ROM], Jutastat [2001].

32. On trouve aussi sur le CD-ROM The State Trials New Series, ed. John MACDONELL, 8 vols., Londres, 1888.

33. Papers of the London Corresponding Society [CD-ROM]: Microform Academic Publishers [n.d]. On trouve les Papers sur trois disques, qui sont constitués des British Library Additional Manuscripts suivants : 27,808; 27,811 - 27,815; 35,142 - 35,143.

34. Mary THALE (ed.), Selections from the Papers of the London Corresponding Society, Cambridge, 1983.

35. Michael T. DAVIS (ed.), London Corresponding Society, 6 vols., Londres, 2002.

36. Mary THALE (ed.), The Autobiography of Francis Place (1771-1854), Cambridge, 1972; «Memoir of Thomas Hardy» in David VINCENT (ed.), Testaments of Radicalism: Memoirs of Working-Class Politicians 1790-1885, Londres, 1977, pp. 31-102.

37. E.P. THOMPSON, The Making of the English Working Class, Harmondsworth, 1968 (1963), p. 162.

38. John GALE JONES, Sketch of a Political Tour through Rochester, Chatham, Maidstone, Gravesend, \&c., ed. Philip MaCDOUGALL, Rochester, 1997.

39. Gregory CLAEYS (ed.), The Politics of English Jacobinism: Writings of John Thelwall, University Park, Pennsylvania, 1995.

40. On trouve dans ce recueil : des extraits du journal The Tribune, où beaucoup de ses conférences publiques étaient reproduites, ainsi que le texte intégral de The Natural and Constitutional Right of Britons (1795); Sober Reflections on the Seditious and Inflammatory Letter of the Rt. Hon. Edmund Burke (1796); et The Rights of Nature (1796).

41. H.T. DICKINSON (ed.), The Political Works of Thomas Spence, Newcastle upon Tyne, 1982; Pig's Meat: Selected Writings of Thomas Spence, Radical and Pioneer Land Reformer, Nottingham, 1982.

42. Michael SCRIVENER (ed.), Poetry and Reform: Periodical Verse from the English Democratic Press 1792-1824, Detroit, 1992.

43. John BARRELl (ed.), Exhibition Extraordinary!! Radical Broadsides of the Mid 1790s, Nottingham, 2001.

44. David BInDman, The Shadow of the Guillotine: Britain and the French Revolution, Londres, 1989, en particulier les pages : 190-206.

45. The Prison Diary (16 May - 22 November 1794) of John Horne Tooke, ed. A.V. BEEDELL and A.D. HARVEY, Leeds, 1995.

46. Michael T. DAVIS, Iain MCCALMAN et Christina PAROLIN (ed.), Newgate in Revolution: An Anthology of Radical Prison Literature in the Age of Revolution, Londres, 2005. On trouve en particulier dans ce 
recueil des textes de James Parkinson, James Lawrence, Thomas Lloyd, Sampson Perry et William Hodgson.

47. Robert RIX (ed.), "A Political Dictionary Explaining the True Meaning of Words'» by Charles Pigott, Aldershot, 2004.

48. Penelope J. CORFIELD et Chris EVANS (ed.), Youth and Revolution in the 1790s, Stroud, 1996.

49. Le sermon fut publié sous le titre : A Discourse on the Love of Our Country (1790). Il a été deux fois réédité dans des éditions modernes, l'une publiée par the National Library of Wales en 1989, et l'autre en 1992 chez Woodstock Books, dans la collection «Revolution and Romanticism».

50. The Correspondence of Richard Price, ed. W. Bernard PEACH and D.O. THOMAS, 3 vols., Durham, NJ and Cardiff, 1983-1994. Le volume 3 couvre la période 1786-1791.

51. Biographie de M. Wollstonecraft par son mari, W. Godwin, parue en 1798.

52. The Political and Philosophical Writings of William Godwin, ed. Pamela CLEMIT, Martin FITZPATRICK and Mark PHILP, 7 vols., Londres, 1993; The Works of Mary Wollstonecraft, ed. Janet TODD and Marlyn BUTLER, 7 vols., Londres, 1989. Il existe aussi The Collected Novels and Memoirs of William Godwin, ed. Pamela CLEMIT, Maurice HINDLE and Mark PHILP, 8 vols., Londres, 1992. Godwin's Political Justice est disponible dans la collection de Woodstock Books «Revolution and Romanticism» ed. Jonathan WORDSWORTH, Oxford, 1992. W. GODWIN, Memoirs of the author of the Vindication of the Rights of Woman, Londres, 1798, est disponible dans de nombreuses éditions, notamment : A Short Residence in Sweden, and Denmark. Mary Wollstonecraft; And Memoirs of the Author of the Rights of Woman. William Godwin, ed. Richard HoLMES, Harmondsworth, 1987 ; Memoirs of Wollstonecraft, ed. Jonathan WORDSWORTH, Oxford, 1993; et Memoirs of the Author of A Vindication of the Rights of Woman, ed. Pamela CLEMIT et Gina Luria WALKER, Peterborough, 2001. On trouve A Vindication of the Rights of Woman de M. Wollstonecraft dans de nombreuses éditions récentes : chez Penguin Classics, ed. Miriam BRODY, Harmondsworth, 1982 ; chez Everyman Classics publié par Dent, ed. Mary WARNOCK, Londres, 1985 ; chez Norton Critical Edition, ed. Carol H. POSTON, New York, 1988 ; enfin chez Cambridge University Press, ed. Sylvana TOMASELLI, Cambridge, 1995.

53. P.A. BROWN, The French Revolution in English History, 1918; rééd., Londres, 1965 ; G.S. VEITCH, The Genesis of Parliamentary Reform, 1913; rééd., Londres, 1965.

54. The Making of the English Working Class a d'abord été publié en anglais en 1963. Il a fallu attendre 1988 pour avoir une traduction française, chez Gallimard-le Seuil.

55. Carl B. CONE, The English Jacobins: Reformers in Late 18th Century England, New York, 1968; Gwyn A. Williams, Artisans and Sans-Culottes: Popular Movements in France and Britain during the French Revolution,1968; reprinted, Londres, 1989.

56. Albert GOODWIN, The Friends of Liberty: The English Democratic Movement in the Age of the French Revolution, Cambridge, Mass., 1979.

57. Voir J.C.D. CLARK, The Language of Liberty 1660-1832: Political Discourse and Social Dynamics in the Anglo-American World, Cambridge, 1994.

58. H.T. DICKINSON, British Radicalism and the French Revolution 1789-1815, Oxford, 1985; H.T. DiCKINSON, The Politics of the People in Eighteenth-Century Britain, Londres, 1995.

59. Jennifer MORI, Britain in the Age of the French Revolution 1785-1820, Harlow, 2000 ; Michael J. TURNER, British Politics in the Age of Reform, Manchester, 1999; Michael J. TURNER, The Age of Unease: Government and Reform in Britain, 1782-1832, Stroud, 2000.

60. Britain and Revolutionary France: Conflict, Subversion and Propaganda, ed. Colin JONES, Exeter, 1983, en particulier les pages : 40-69.

61. J. Ann HONE, For the Cause of Truth: Radicalism in London 1796-1821, Oxford, 1982.

62. Ray HEMMINGS, Liberty or Death: Early Struggles for Parliamentary Democracy, Londres, 2000.

63. Jenny GRAHAM, The Nation, the Law and the King: Reform Politics in England, 1789-1799, 2 vols., Lanham, Maryland, 2000.

64. Goodwin, The Friends of Liberty, op. cit., en particulier les pages : 136-170. 
65. Edwin JAGGARD, Cornwall Politics in the Age of Reform, Woodbridge, 1999.

66. John STEVENSON, Artisans and Democrats: Sheffield in the French Revolution, 1789-97, Londres, 1989. Sur la Sheffield SCI, voir F.K. DONNELLY, "The Foundation of the sheffield Society for Constitutional Information», Labour History Review, 56 (1991), pp. 51-53.

67. David V. ERDMAN, Commerce des Lumières: John Oswald and the British in Paris, 1790-1793, Columbia, 1986.

68. George RUDÉ, Protest and Punishment: The Story of the Social and Political Protesters Transported to Australia, 1788-1868, Oxford, 1978.

69. Richard J. twomey, Jacobins and Jeffersonians: Anglo-American Radicalism in the United States, 1790-1820, New York, 1989; Richard J. TWOMEY, «Jacobins and Jeffersonians: Anglo-American Radical Ideology, 1790-1810», in Margaret C. ЈАСОВ and James R. ЈАСОВ (ed.), The Origins of AngloAmerican Radicalism, New Jersey, 1984, pp. 313-328.

70. Michael DUREY, Transatlantic Radicals and the Early American Republic, Kansas, 1997.

71. Whitney R.D. JONES, David Williams: The Anvil and the Hammer, Cardiff, 1986.

72. Pour une étude de qualité sur la pensée de Priestley, voir Jack FRUCHTMAN, The Apocalyptic Politics of Richard Price and Joseph Priestley, Philadelphia, 1983.

73. Jenny GRAHAM, Revolutionary in Exile: The Emigration of Joseph Priestley to America, 1794-1804, Philadelphia, 1995.

74. Joseph Priestley and Birmingham, ed. Malcolm DICK, Studley, 2005.

75. Robert E. SCHOFIELD, The Enlightened Joseph Priestley: A Study of His Life and Work from 1773 to 1804, University Park, Pennsylvania, 2004.

76. C. KEGAN PAUL, William Godwin: His Friends and Contemporaries, 2 vols., Londres, 1876.

77. Ian WARD, «A Love of Justice: The Legal and Political Thought of William Godwin», The Journal of Legal History, 25 (2004), p. 1.

78. Mark PHILP, Godwin's Political Justice, Londres, 1986.

79. Don LOCKE, A Fantasy of Reason: The Life and Thought of William Godwin, Londres, 1980; Peter MARSHALL, William GODWIN, New Haven, 1984.

80. William ST CLAIR, The Godwins and the Shelleys: The Biography of a Family, Londres, 1989.

81. Gary KELLY, Revolutionary Feminism: The Mind and Career of Mary Wollstonecraft, Basingstoke, 1992; Janet TODD, Mary Wollstonecraft: A Revolutionary Life, Londres, 2000; Diane JACOBS, Her Own Woman: The Life of Mary Wollstonecraft, Londres, 2001; Claudia L. JOHNSON (ed.), The Cambridge Companion to Mary Wollstonecraft, Cambridge, 2002; Barbara TAYLOR, Mary Wollstonecraft and the Feminist Imagination, Cambridge, 2003; Lyndal GORDON, Mary Wollstonecraft: A New Genius, Londres, 2005.

82. Helen BRAITHWAITE, Romanticism, Publishing and Dissent: Joseph Johnson and the Cause of Liberty, Basingstoke, 2003.

83. Jack FRUCHTMAN, Thomas Paine: Apostle of Freedom, New York, 1994; John KEANE, Tom Paine: A Political Life, Londres, 1995; Trevor GRIFFITHS, These Are The Times: A Life of Thomas Paine, Londres, 2004.

84. Sur l'ampleur des erreurs dans le livre de Keane, voir History, 81 (1996), pp. 228-237.

85. Kenneth Johnston, The Hidden Wordsworth: Poet, Lover, Rebel, Spy, Londres et New York, 1998. C'est Michael Durey qui a le premier mis le doigt sur les erreurs de K. Johnson : « The Spy Who Never Was », Times Literary Supplement, 10 March 2000, pp. 14-15. Lorsque le livre de K. Johnson est ressorti en 2000, la référence à « l'espion » a été supprimée dans le sous-titre, en réponse aux critiques de M. Durey. Voir John MULLAN, «Bumming and Booing», London Review of Books, 5 April 2001; et la lettre de K. Johnston à la London Review of Books, 24 mai 2001.

86. Voir Michael T. DAVIS, «"That Odious Class of Men Called Democrats”: Daniel Isaac Eaton and the Romantics, 1794-95», History, 84 (1999), pp. 74-92. 
87. Nicholas ROE, Wordsworth and Coleridge: The Radical Years, Oxford, 1988.

88. Christina BEWLEY and David BEWLEY, Gentleman Radical: A Life of John Horne Tooke, Londres, 1998.

89. David ALEXANDER, Richard Newton and English Caricature in the 1790s, Manchester, 1998.

90. Philip HARLING, «Equipoise Regained? Recent Trends in British Political History, 1790-1867», The Journal of Modern History, 75 (2003), pp. 890-891.

91. Voir Mark PHILP, «English Republicanism in the 1790s», The Journal of Political Philosophy, 6 (1998), pp. 235-262.

92. Voir Bernard BAILYN, The Ideological Origins of the American Revolution, Cambridge, Mass., 1976; Herbert BUTTERFIELD, George III, Lord North, and the People, 1779-1780, Londres, 1949; et Ian R. CHRISTIE, Myth and Reality in Late Eighteenth-Century British Politics, Londres, 1970.

93. Carla HAY, James Burgh, Spokesman of Reform in Hanoverian England, Washington, 1979, pp. 104-105.

94. Voir J.G.A. Рососк, The Machiavellian Moment: Florentine Political Thought and the Atlantic Republican Tradition, Princeton, N.J., 1975. Voir aussi J.G.A. Рососк, Virtue, Commerce and History: Essays on Political Thought and History, Chiefly in the Eighteenth Century, Cambridge, 1985.

95. Isaac KRAMNICK, Republicanism and Bourgeois Radicalism: Political Ideology in Late EighteenthCentury England and America, Ithaca, N.Y., 1990; Isaac KRAMNICK, «Republican Revisionism Revisited», American Historical Review, 87 (1982), pp. 629-644.

96. KRAMNICK, Republicanism and Bourgeois Radicalism, op. cit., p. 4.

97. H. T. DICKINSON, The Politics of the People in Eighteenth-Century Britain, op. cit., p. 179.

98. Gregory CLAEYS, Thomas Paine: Social and Political Thought, Boston and Londres, 1989.

99. Michael SCRIVENER, Seditious Allegories: John Thelwall and Jacobin Writing, University Park, Pennsylvania, 2001.

100. Robert HOLE, Pulpits, Politics and Public Order in England, 1760-1832, Cambridge, 1989.

101. Eileen GROTH LYON, Politicians in the Pulpit: Christian Radicalism in Britain from the Fall of the Bastille to the Disintegration of Chartism, Aldershot, 1999.

102. Stuart ANDREWS, Unitarian Radicalism: Political Rhetoric, 1770-1814, Basingstoke, 2003.

103. H.T. DICKINSON, Liberty and Property: Political Ideology in Eighteenth-Century Britain, Londres, 1977, p. 1.

104. Pour une discussion de qualité sur ces questions, et sur l'impact des cultural studies sur l'histoire du radicalisme, voir : Alex BENCHIMOL, «Remaking the Romantic Period: Cultural Materialism, Cultural Studies and the Radical Public Sphere», Textual Practice, 19 (2005), pp. 51-70. 105. Craig CALHoun, The Question of Class Struggle: Social Foundations of Popular Radicalism during the Industrial Revolution, Chicago, 1982.

106. Iain mCCALMAN, Radical Underworld: Prophets, Revolutionaries and Pornographers in London, 1795-1840, Cambridge, 1988.

107. Cette notion d'« études plébéiennes » est empruntée à un compte rendu de Anne JANOwiTz in Studies in Romanticism, 32 (1993), pp. 297-303.

108. David WORRALl, Radical Culture: Discourse, Resistance and Surveillance, 1790-1820, Hemel Hempstead, 1992; Jon MEE, Dangerous Enthusiasm: William Blake and the Culture of Enthusiasm in the 1790s, Oxford, 1992.

109. Geoff ELEY, «Nations, Publics, and Political Cultures: Placing Habermas in the Nineteenth Century", in Craig CalHoun (ed.), Habermas and the Public Sphere, , Cambridge, Mass., 1992, pp. 318-331; Terry EAGLETON, The Function of Criticism, from the Spectator to Post-Structuralism, Londres, 1984; Jon KLANCHER, The Making of English Reading Audiences, 1790-1832, Madison, 1987.

110. Kevin Gilmartin, Print Politics: The Press and Radical Opposition in Early Nineteenth-Century England, Cambridge, 1996.

111. Id., p. 27. 
112. Stuart ANDREWS, The British Periodical Press and the French Revolution, 1789-99, Basingstoke, 2000, en particulier les pages 124-137.

113. Paul KEEN, The Crisis of Literature in the 1790s: Print Culture and the Public Sphere, Cambridge, 1999. Voir aussi Andrew MCCANN, Cultural Politics in the 1790s: Literature, Radicalism and the Public Sphere, Basingstoke, 1998.

114. Robert M. MANIQUIS (ed.), British Radical Culture of the 1790s, San Marino, 2002.

115. Marcus wood, Radical Satire and Print Culture 1790-1822, Oxford, 1994.

116. H.T. DICKINSON, Caricatures and the Constitution, 1760-1832, Cambridge, 1985; Tamara L. HUNT, Defining John Bull: Political Caricature and National Identity in Late Georgian England, Aldershot, 2003.

117. James EPSTEIN, Radical Expression: Political Language, Ritual and Symbol in England, 1790-1850, New York, 1994; James EPSTEIN, In Practice: Studies in the Language and Culture of Popular Politics in Modern Britain, Stanford, 2003.

118. Voir James EPSTEIN et David KARR, «Playing at Revolution: British "Jacobin" Performance», Journal of Modern History (à paraître). Je remercie James Epstein de m'avoir donné une copie de cet article.

119. James EPSTEIN, "“Our Real Constitution": Trial Defence and Radical Memory in the Age of Revolution", in James VERNON (ed.), Re-Reading the Constitution: New Narratives in the Political History of England's Long Nineteenth Century, Cambridge, 1996, pp. 22-51; James EPSTEIN, «"Equality and No King": Sociability and Sedition: The Case of John Frost», in Gillian RUSSELL and Clara TUITE (ed.), Romantic Sociability: Social Networks and Literary Culture in Britain 1770-1840, Cambridge, 2002, pp. 43-61; John BARRELL, «Coffee-House Politicians», Journal of British Studies, 43 (2004), pp. 206-232. Voir aussi John BARRELL, «"An Entire Change of Performances?” The Politicisation of Theatre and the Theatricalisation of Politics in the Mid 1790s», Lume: Selected Proceedings from the Canadian Society for Eighteenth-Century Studies, 17 (1998), pp. 11-50.

120. J. BREWER et J. STYLES, An Ungovernable People: The English and their Law in the Seventeenth and Eighteenth Centuries, Londres, 1983; Ian GILMOUR, Riot, Risings and Revolution: Governance and Violence in Eighteenth-Century England, Londres, Pimlico, 1992.

121. John вонSTEDT, Riots and Community Politics in England and Wales 1790-1810, Cambridge, Mass., 1983.

122. J.M. Golby et A.W. PURdue, The Civilisation of the Crowd: Popular Culture in England 1750-1900, Stroud, 1999; George RUDÉ, The Crowd in History, Londres, 1964; Nicholas ROGERS, Crowds, Culture and Politics in Georgian Britain, Oxford, 1998.

123. Charles TILly, Popular Contention in Great Britain 1758-1834, Cambridge, Mass., 1995.

124. Roger WELLS, Wretched Faces: Famine in Wartime England 1793-1803, Gloucester, 1998.

125. Ian GILMOUR, Riot, Risings and Revolution, Londres, Pimlico, 1992.

126. Ian R. CHRISTIE, Stress and Stability in Late Eighteenth-Century Britain: Reflections on the British Avoidance of Revolution, Oxford, 1984.

127. Edward ROYLE, Revolutionary Britannia? Reflections on the Threat of Revolution in Britain 1789-1848, Manchester and New York, 2000, p. 10.

128. Voir Steve Poole, The Politics of Regicide in England, 1760-1850, Manchester et New York, 2000; Frank PROCHASKA, The Republic of Britain, 1760 to 2000, Londres, 2000, pp. 1-32; et Anthony TAYLOR, «Down With The Crown»: British Anti-Monarchism and Debates about Royalty since 1790, Londres, 1999.

129. Malcolm I. тномIS and Peter ноLт, Threats of Revolution in Britain 1789-1848, Basingstoke, 1977.

130. I. MCCALMAN, Radical Underworld.

131. Roger WELLS, Insurrection: The British Experience 1795-1803, Gloucester, 1983.

132. Clive EMSLEY, «An Aspect of Pitt's Terror: Prosecutions for Sedition during the 1790s», Social History, 6 (1981), pp. 155-184; Clive EMSLEY, «Repression, “Terror" and the Rule of Law during the Decade of the French Revolution», English Historical Review, 100 (1985), pp. 801-825; et Philip 
HARLING, «The Law of Libel and the Limits of Repression, 1790-1832», The Historical Journal, 44 (2001), pp. 107-134.

133. Michael LoBban, «From Seditious Libel to Unlawful Assembly: Peterloo and the Changing Face of Political Crime c. 1770-1820», Oxford Journal of Legal Studies, 10 (1990), pp. 307-352. Pour un débat sur la répression du commerce des livres, voir Donald THOMAs, «Press Prosecutions of the Eighteenth and Nineteenth Centuries: The Evidence of the Kings' Bench Indictments», The Library, 32 (1977), pp. 315-332 ; John FEATHER, «The English Book Trade and the Law 1695-1799», Publishing History, 12 (1982), pp. 51-75 ; Alan воотн, «"The Memory of the Liberty of the Press": The Suppression of Radical Writing in the 1790s», in Paul HYLAND et Neill SAMmELLs (ed.), Writing and Censorship in Britain, Londres, 1992, pp. 107-122 ; Jon MEE, "“Examples of Safe Printing": Censorship and Popular Radical Literature in the 1790s», Essays and Studies, 46 (1993), 81-95 ; Ian HAYwood, The Revolution in Popular Literature: Print, Politics and the People, 1790-1860, Cambridge, 2004, pp. 81-111.

134. Je suis actuellement engagé dans un projet sur les « procès politiques à l'ère de la contrerévolution : performance et discours ", qui débouchera sur un livre.

135. Alan WHARAM, The Treason Trials of 1794, Leicester, 1992.

136. John BARRELL, Imagining the King's Death. Figurative Treason, Fantasies of Regicide 1793-1796, Oxford, 2000.

137. EPSTEIN, “"Our Real Constitution": Trial Defence and Radical Memory in the Age of Revolution»; Michael T. DAVIS, «"The Impartial Voice of Future Times Will Rejudge Your Verdict”: Discourse and Drama in the Trials of the Scottish Political Martyrs of the 1790s», in Joanne PAISANA (ed.), Hélio Osvaldo Alves: O Guardador de Rios, Braga, 2005, pp. 65-78. Voir également Michael T. DAVIS, «"I Can Bear Punishment" : Daniel Isaac Eaton, Radical Culture and the Rule of Law, 1793-1812», Criminal Justice History, 18 (2003), pp. 89-106.

\section{RÉSUMÉS}

Bien que le radicalisme anglais soit antérieur à la Révolution française, il ne fait aucun doute que les événements spectaculaires des années 1790 ont eu une profonde influence sur le développement et le déclin du mouvement radical britannique. Beaucoup d'études ont été consacrées à ce phénomène et cet article fait le point sur les travaux qui, fondés sur les sources primaires et imprimées, s'attachent aux situations générales et régionales, aux liens internationaux, aux biographies des dirigeants radicaux, à la culture et à l'idéologie radicales, à la peur de la révolution et à la répression du radicalisme.

English Radicalism and the French Revolution. Although English radicalism originated before the French Revolution there can be no doubt that the dramatic events in France from 1789 onwards had a profound effect on the rise and fall of English radicalism in the 1790s. There has been much recent research on this topic and this essay surveys and assesses the work produced on the primary sources and printed propaganda, the general and regional studies, the overseas links, the biographies of leading radicals, radical ideology and radical culture, the threat of revolution and the government suppression of radicalism. 
INDEX

Mots-clés : Radicalisme, idéologie, répression, clubs et sociétés

\section{AUTEUR}

\section{MICHAEL DAVIS}

The University of Queensland, Centre for the History of European Discourses (CHED), ForganSmyth Level 5, Brisbane, Qld 4072 Australia 